\title{
Reversal of dysthyroid optic neuropathy following orbital fat decompression
}

\author{
Michael Kazim, Stephen L Trokel, Golge Acaroglu, Alexandra Elliott
}

\begin{abstract}
Aims-To document the successful treatment of five patients with dysthyroid optic neuropathy by orbital fat decompression instead of orbital bone decompression after failed medical therapy.

Methods-Eight orbits of five patients with dysthyroid optic neuropathy were selected for orbital fat decompression as an alternative to bone removal decompression. Treatment with systemic corticosteroids and/or orbital radiotherapy was either unsuccessful or contraindicated in each case. All patients satisfied clinical indications for orbital bone decompression to reverse the optic neuropathy. High resolution computerised tomographic (CT) scans were performed in all cases and in each case showed signs of enlargement of the orbital fat compartment. As an alternative to bone decompression, orbital fat decompression was performed on all eight orbits.
\end{abstract}

Results-Orbital fat decompression was performed on five patients (eight orbits) with optic neuropathy. Optic neuropathy was reversed in all cases. There were no cases of postoperative diplopia, enophthalmos, globe ptosis, or anaesthesia. All patients were followed for a minimum of 1 year.

Conclusions-In a subset of patients with an enlarged orbital fat compartment and in whom extraocular muscle enlargement is not the solitary cause of optic neuropathy, fat decompression is a surgical alternative to bony decompression.

(Br F Ophthalmol 2000;84:600-605)

Ophthalmo

Edward S Harkness

Eye Institute,

Columbia-Presbyterian

Medical Center, New

York, USA

M Kazim

S L Trokel

G Acaroglu

A Elliott

Department of

Surgery,

Columbia-Presbyterian

Medical Center, New

York, USA

M Kazim

Correspondence to: Michael Kazim, MD, Edward S Harkness Eye Institute, 635 West 165 th Street, New York, NY 10032 USA

mk48@columbia.edu

Accepted for publication 10 January 2000
Dysthyroid optic neuropathy has been effectively treated with various combinations of radiotherapy. high dose corticosteroids, radiotherapy, and surgical enlargement of the orbital cavity by removal of one or more orbital walls. When used alone, corticosteroids generally require long courses of high dose therapy to prevent relapses. ${ }^{56}$ Adverse systemic effects limit long term corticosteroid treatment. Orbital radiotherapy (2000 cGy) has been shown to be at least as effective as high dose oral steroid therapy (80-120 $\mathrm{mg}$ prednisone) in relieving optic neuropathy. ${ }^{7-9}$

When medical therapy fails or is contraindicated, reversal of optic neuropathy has traditionally been achieved by enlarging the orbital space via surgical bone decompression. This is believed to relieve pressure on the apical optic nerve by permitting the distended orbital soft tissues to expand into surrounding spaces. ${ }^{10-13}$ Historically, fat decompression had been restricted in its clinical indications to reducing exophthalmos, eliminating orbital pain, and reducing congestion. ${ }^{14-16}$ We postulate that restoration of normal orbital volume, by removal of orbital fat, decreases orbital pressure sufficiently to allow reversal of optic neuropathy in selected cases.

\section{Materials and methods}

Five patients with dysthyroid optic neuropathy who had failed to respond to orbital radiotherapy or corticosteroids and met the clinical criteria for bone decompression, were treated by orbital fat decompression in a manner previously described by the authors. ${ }^{15}$

Preoperatively, the patients received $10 \mathrm{mg}$ of Decadron (dexamethasone, MSD) and $1 \mathrm{~g}$ of cephazolin. A lid crease incision was made in the medial third of the upper lid and a subciliary incision in the lower lid. The orbital septum was opened and prolapsing orbital fat was removed using blunt surgical dissection and unipolar electrocautery. The use of ribbon retractors enabled dissection deep into the orbit. Traction on the anterior orbital fat was used to gain access to the intraconal fat. The fat was not cross clamped before excision. Electrocautery was used to cut fat and coagulate directly visualised blood vessels. On average, 3-6 $\mathrm{ml}$ of fat were removed through the lower compartment and $2 \mathrm{ml}$ through the superior fat compartment. The surgical beds were irrigated with saline and inspected for bleeding. The skin incision was closed with a 6-0 nylon suture that was removed on the fifth postoperative day. The wounds were dressed with antibiotic ointment. The patients were observed for 24 hours in the hospital for the development of postoperative haemorrhage and treated with ice packs. 


\section{Results}

CASE 1

A 61 year old man developed orbitopathy 7 months before referral for evaluation of deteriorating visual acuity in the left eye despite treatment with $70 \mathrm{mg}$ of prednisone daily. The initial examination revealed visual acuity of 20/25 right eye, 20/40 left eye. He identified 12 of 12 Ishihara colour plates with his right eye and nine of 12 with the left. No relative afferent pupillary defect (RAPD) was detected and the optic discs were normal in appearance. Hertel measurements were $22.5 \mathrm{~mm}$ right eye, and $24.5 \mathrm{~mm}$ left. Upgaze was impaired bilaterally, more on the left. CT images revealed a moderately enlarged left medial rectus and slightly enlarged inferior recti bilaterally. Bilateral orbital radiotherapy (2000 cGy in 10 fractions) was instituted. He received $40 \mathrm{mg}$ of prednisone daily, which was reduced over a period of 3 weeks to $30 \mathrm{mg}$ four times daily and ultimately discontinued at 6 weeks. At that time, visual acuity was $20 / 25$ right eye and $20 / 30$ left eye; colour vision was $12 / 12$ right eye and $11 / 12$ left eye.

Six weeks after completion of radiotherapy and 2 weeks after cessation of corticosteroids, visual acuity deteriorated to 20/50 left eye, colour vision was $7 / 12$, and a moderate left RAPD was present. A left inferior altitudinal field defect was identified by threshold perimetry, and ocular rotations were more limited. Left exophthalmos had increased to $26.5 \mathrm{~mm}$. Further enlargement of medial, inferior, superior recti and both superior oblique muscles was seen on CT images, as well as additional crowding at the orbital apices and straightening of the left optic nerve, a sign of enlargement of the orbital fat compartment (Fig 1A, B).

The patient underwent left orbital fat decompression. He received $40 \mathrm{mg}$ prednisone four times daily postoperatively. On the third postoperative day he identified eight of $12 \mathrm{col}-$ our plates. Five weeks postoperatively, left visual acuity was $20 / 30$, colour vision was $12 / 12$, and there was no pupil abnormality. Prednisone was tapered and discontinued by 3 months after surgery. Visual acuity 15 months after surgery was $20 / 20$ right eye and 20/25 left eye. Colour vision, pupil reactivity, and visual fields were normal. Exophthalmos was reduced to $21 \mathrm{~mm}$ left eye, and ocular rotations were limited in left upgaze only.

CASE 2

A 27 year old woman with dysthyroid orbitopathy was referred for deteriorating vision. Visual acuity was 20/50 right eye and 20/100 left. She identified 2/12 Ishihara colour plates with the right eye and $1 / 12$ with the left. Optic discs appeared normal, no RAPD was detected, but visual fields were constricted bilaterally. Hertel measurements were $22 \mathrm{~mm}$ right eye and 23 $\mathrm{mm}$ left eye with bilaterally limited abduction. The CT scan revealed bilateral medial and inferior rectus enlargement and crowding of the orbital apices. Increased orbital fat volume and density were identified (Fig 2A). She received $80 \mathrm{mg}$ prednisone daily, and orbital
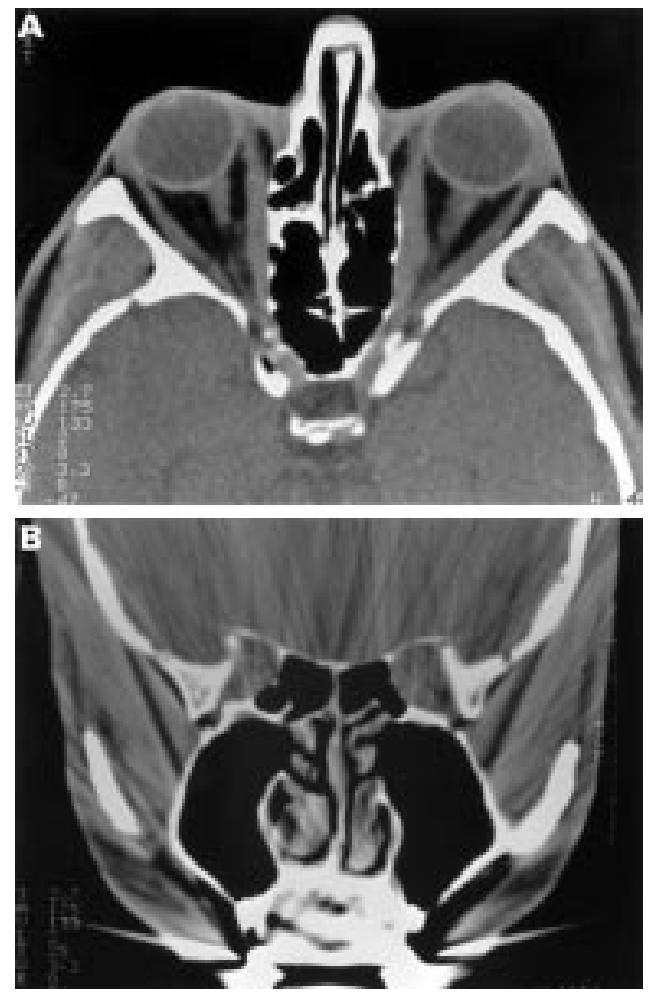

Figure 1 Preoperative CT scan of patient 1. Axial mid-orbit $(A)$ and coronal apical section $(B)$. Note apical enlargement of the medial recti more significant on the left.

radiotherapy was begun (2000 cGy in 10 fractions). During the course of radiotherapy, her visual acuity fluctuated, colour vision deterio-
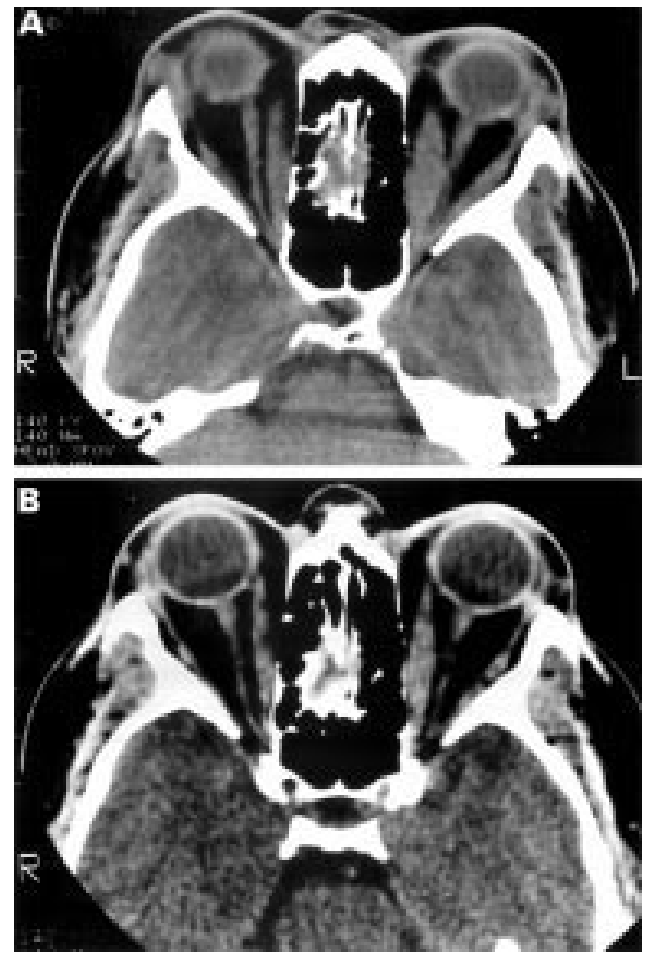

Figure 2 (A) Axial CT scan of patient 2 before radiotherapy. Note enlarged medial recti worse left than right. (B) Axial CT scan of patient 2, after completion of radiotherapy. Note reduction in size of medial recti, absence of apical crowding of the optic nerve, and straightening of the optic nerve shadows. 
rated to no colour perception bilaterally, and there was minimal improvement in the visual fields.

Three days after the completion of radiotherapy the patient experienced bilateral vision loss. Acuity was 20/200 right eye and 20/400 left eye, she had no colour vision, and visual fields were severely constricted. Bone decompression was considered. The CT scan was repeated and revealed normal paranasal sinuses, and significant shrinkage, to near normal diameter, of the previously swollen extraocular muscles without apical crowding. The optic nerves appeared straightened, lacking the usual sinusoidal curve (Fig 2B).

The patient was hospitalised and dexamethasone was administered intravenously (10 mg every 6 hours). Twenty four hours after admission her visual acuity was counting fingers in both eyes. Orbital fat decompression was performed bilaterally.

Improvement of visual function was detected within hours after surgery. She identified seven of 12 colour plates with both eyes on the evening of surgery. On the second postoperative day, she identified 12 of 12 colour plates with both eyes and her visual acuity was $20 / 60$ right eye and 20/50 left eye.

Steroids were gradually tapered and discontinued over 5 weeks. By the fourth postoperative month, her acuity was $20 / 20$ in both eyes. Colour vision and visual fields were normal. Hertel measurements were $18 \mathrm{~mm}$ right and $19 \mathrm{~mm}$ left.

CASE 3

A 67 year old man developed dysthyroid orbitopathy 6 months before referral for treatment of optic neuropathy. He had been previously treated with corticosteroids that produced acute uncontrollable gastrointestinal bleeding. A ventral hernia further complicated the emergency abdominal surgery. Orbital radiotherapy was delivered at another institution without improvement in vision. Bone decompression of the left orbit was recommended by the referring neuro-ophthalmologist.

Visual acuity was 20/30 right eye and 20/60 left eye. He recognised 11 of 12 Ishihara colour plates with the right eye and 7/12 with the left. Optic discs were normal. Visual fields showed generalised reduction in sensitivity and inferior altitudinal field loss in the left eye. A CT scan revealed crowding of the orbital apices by enlarged medial, inferior, and superior rectus muscles bilaterally (Fig 3A, B). Vertical ocular rotations were symmetrically limited. Hertel measurements were $22 \mathrm{~mm}$ right and $24 \mathrm{~mm}$ left.

The patient was monitored without corticosteroid treatment. Three weeks later, colour perception had deteriorated to 6 of 12 in the left eye and the visual field loss persisted. Orbital fat decompression of the left orbit was performed in an attempt to restore normal orbital volume dynamics without the need for corticosteroids or more complex bone decompression surgery.

Three days postoperatively, colour vision improved to 9 of 12 Ishihara plates. Two weeks
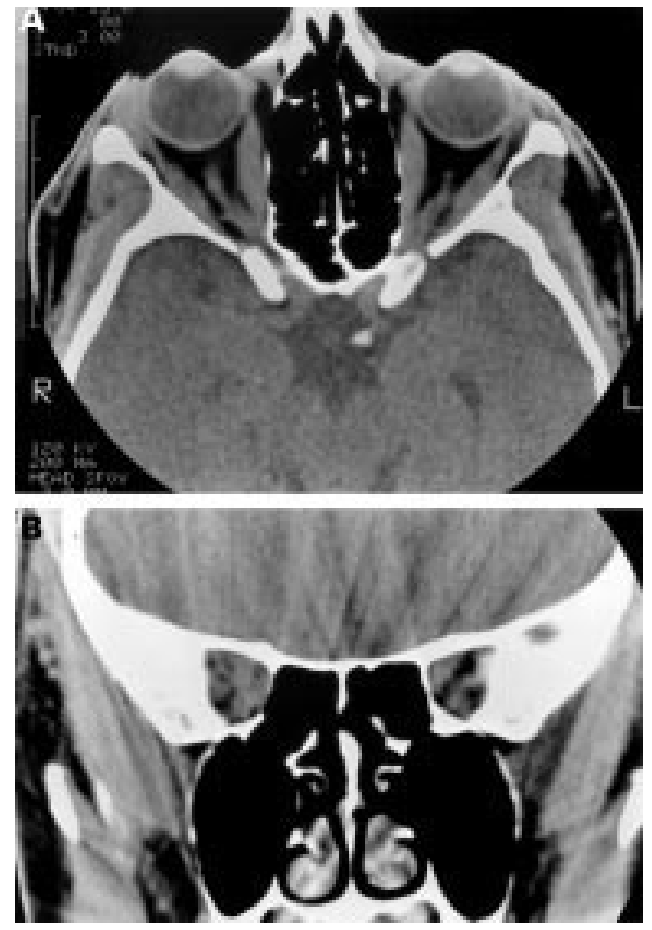

Figure 3 Preoperative axial (A) and coronal (B) CT scan of patient 3. Note bilateral enlargement of medial, inferior, and superior recti.

later his acuity was $20 / 40$ and the visual field had improved. Two months after surgery, his vision stabilised at 20/30, he continued to identify 9 of 12 Ishihara colour plates. He was orthophoric and the visual field continued to improved. Hertel readings were $22 \mathrm{~mm}$ bilaterally.

\section{CASE 4}

A 61 year old woman presented with bilateral dysthyroid optic neuropathy with substantial congestive orbital signs. Visual acuity was 20/25 right eye and 20/30 left eye with normal colour vision. Venous engorgement was present in both fundi and optic disc swelling was present in the left eye. A left RAPD was present. Visual fields exhibited bilateral generalised depression of sensitivity and constriction, more severe on the left. Hertel readings were $20 \mathrm{~mm}$ right and $24.5 \mathrm{~mm}$ left. Elevation and depression was limited bilaterally, more so on the left. CT imaging showed marked bilateral enlargement of the medial, inferior, and superior rectus muscles and moderate enlargement of the left lateral rectus. An increase in the volume of the orbital fat compartment was also apparent. Radiotherapy (2000 cGy) was delivered to both orbits and she received $40 \mathrm{mg}$ prednisone four times a day

One week after completion of the radiotherapy, the optic disc swelling and the congestive signs were significantly relieved. Steroids were tapered and discontinued in 8 weeks.

Four months later the orbitopathy recurred and she complained of transient visual obscurations in the right eye. There was massive right optic disc swelling and mild optic disc swelling in the left eye. Visual acuity was $20 / 50$ right eye and 20/40 left eye. Colour vision was 
not affected. Hertel readings were $24.5 \mathrm{~mm}$ right eye and $25 \mathrm{~mm}$ left eye.

Five months after completion of radiotherapy, she underwent bilateral fat decompression and received supplemental prednisone; $40 \mathrm{mg}$ alternating with $20 \mathrm{mg}$ four times daily.

Three weeks after surgery, the right optic disc swelling was improved and the left optic disc was normal. One month later, prednisone was reduced to $20 \mathrm{mg}$ four times daily, the vision was $20 / 20$ in each eye and colour plate recognition was $11 / 12$ with the right eye and $11 / 12$ with the left. Hertel measurements were $22.5 \mathrm{~mm}$ right eye and 23.5 left eye. Evidence of mild chronic disc swelling persisted for 2 months after surgery. Her vision was $20 / 20$ in both eyes with normal discs when prednisone was discontinued in the fourth postoperative month.

CASE 5

A 44 year old woman presented with bilateral dysthyroid optic neuropathy. Visual acuity was $20 / 40$ in both eyes and she was unable to identify any Ishihara colour plates with either eye. No relative afferent pupil defect appreciated. Threshold visual field testing demonstrated bilateral inferior altitudinal paracentral scotomas. Hertel measurements were $24 \mathrm{~mm}$ right eye and $27 \mathrm{~mm}$ left. Ocular rotations were moderately impaired in all fields of gaze in the right eye and more severely in the left. Hyperaemia of the left optic disc was apparent, the right disc was normal. A CT scan demonstrated bilateral fusiform enlargement of all rectus muscles, the orbital fat compartment was unremarkable. She was treated with radiotherapy (2000 cGy) to both orbits and prednisone, $60 \mathrm{mg}$ daily.

Two weeks after completion of the radiotherapy, prednisone was reduced to an alternate day regimen of $40 / 20 \mathrm{mg}$. She identified 11/12 Ishihara colour plates with the right eye and $10 / 12$ with the left. The inferior altitudinal visual field defects had become denser. Attempts to taper the corticosteroids over the subsequent 7 months resulted in recurrence of the optic neuropathy in the left eye as evidenced by loss of colour vision (12/12 right eye and 0/12 left eye), a moderate left afferent pupil defect and further inferior visual field loss. Hertel measurements were $26 \mathrm{~mm}$ right eye and $27 \mathrm{~mm}$ left eye. The patient refused further corticosteroid treatment. Orbital decompression was entertained and a CT scan performed. The rectus muscles remained enlarged with crowding of the orbital apex (Fig $4 \mathrm{~A}, \mathrm{~B})$.

Bone decompression was considered but rejected by the patient. Bilateral fat decompression was performed. Over the following 5 weeks, prednisone was discontinued and visual function improved. Visual acuity (20/20 right eye, 20/25 left eye), colour perception (12/12 in both eyes), and pupil reactions have remained stable over the subsequent year and the field defect has nearly resolved. Hertel measurements are $22 \mathrm{~mm}$ right eye and $23 \mathrm{~mm}$ left eye.
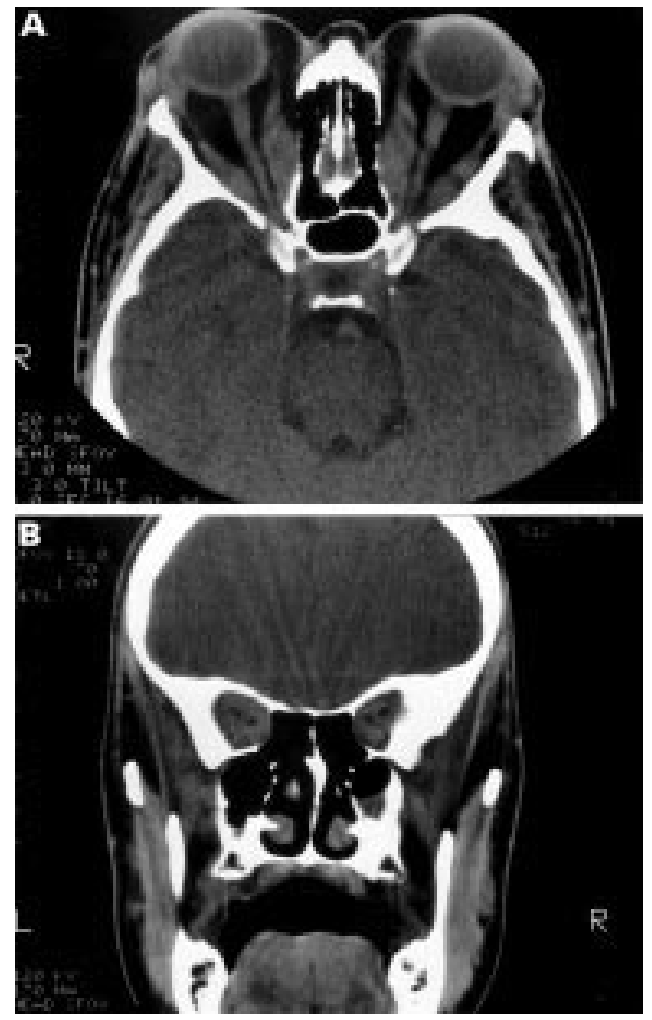

Figure 4 Preoperative axial (A) and coronal (B) CT scan of patient 5. Note bilateral enlargement of all rectus muscles with straightening of the optic nerve shadow and crowding of the orbital apices bilaterally.

\section{Discussion}

The cases presented in this report provoke consideration of the commonly held view that bone decompression is required to treat dysthyroid optic neuropathy. An early theory of dysthyroid optic neuropathy held that an inflammatory neuritis was produced by the generalised orbital inflammation present in the acute phase of the disease. ${ }^{17}{ }^{18}$ This theory, while consistent with the current understanding of optic neuropathy associated with chronic sphenoid sinusitis, ${ }^{19}$ fell out of favour when the study of necropsy specimens in chronic thyroid eye disease failed to demonstrate an inflammatory infiltrate of the optic nerve. ${ }^{20}{ }^{21}$ It should be noted that no histopathological study of an optic nerve with acute dysthyroid optic neuropathy has appeared in the literature. Therefore, the role of inflammatory infiltration of the optic nerve during the acute phase of dysthyroid optic neuropathy remains an open question.

Subsequent theories were developed based on the results of CT images. Early generation CT images of patients with dysthyroid orbitopathy revealed fusiform enlargement of the extraocular muscles associated with crowding at the orbital apex. ${ }^{1-4}$ The anatomical distortion of the muscles was felt to produce impairment of optic nerve function by direct compression. This theory is supported by the gross appearance of orbital specimens from patients with chronic Graves' orbitopathy which have demonstrated compression of the optic nerve at the orbital apex by the massively enlarged extraocular muscles. ${ }^{21}$ Histopatho- 
logical examination has revealed axonal loss, more pronounced in the apical sections of the optic nerve.$^{21}$ Clinical evidence is derived from the dramatic improvement in visual acuity that can occur within hours after bony decompression that relieves the pressure in the crowded orbital apex. ${ }^{1320}$ Furthermore, the visual field defects identified in patients with dysthyroid optic neuropathy are similar to those produced by other orbital mass lesions that compress the optic nerve. ${ }^{4}$

The theory of direct compression of the apical portion of the optic nerve by enlarged extraocular muscles has been used to explain the vast majority of cases for the past two decades. There are, however, cases that feature clinical or radiographic findings that do not fit this model. Three dimensional volumetric analysis of new generation, high resolution orbital CT images have shown that there may be three subpopulations of patients with dysthyroid orbitopathy. ${ }^{2}{ }^{152-26}$ Most commonly seen is the first group that has both increased extraocular muscle (EOM) and fat compartment volumes. The second largest group features increased EOM volume and normal orbital fat compartment volume. The least common population has increased fat compartment volume, normal EOM volume and straightened optic nerves.

It is reasonable to expect that increased soft tissue volume from any source within the confines of the bony orbit will increase orbital pressure. Increased orbital pressure produces variable signs and symptoms that are related to individual differences in orbital and lid anatomy. Among the clinical findings are deep orbital pain, proptosis, impaired motility and, possibly, impaired optic nerve function.

In dysthyroid orbitopathy the degree of orbital soft tissue expansion is not directly proportional to the degree of proptosis because of two anatomical variables. First is fibrosis of the soft tissues (in particular the extraocular muscles and intermuscular septations) which limits anterior displacement of the globe. The other is laxity of the eyelids, tendons, and septum which generally increases with age. Younger patients and those with greater orbital fibrosis will have less proptosis for a given increase in orbital volume, and develop higher orbital pressures. Conversely patients with marked lid laxity and minimal fibrosis of the orbital tissues will show more proptosis and lower orbital pressures because of spontaneous anteriorly directed decompression. It has often been noted that marked proptosis is often absent in patients with optic neuropathy. ${ }^{47}$ Furthermore, the degree of proptosis may not correlate with the severity of neuropathy ${ }^{15}$ or the risk of development of neuropathy. ${ }^{28}$ It may therefore be argued that it is orbital pressure and not proptosis that correlates with optic neuropathy. Measurement of orbital pressure has been limited to qualitative assessments of resistance to retropulsion of the globes, a crude assessment. No device has been proved to accurately and reproducibly measure intraorbital pressure..$^{29} 30$ A technique for measuring orbital tension and orbital compartment compliance using an orbital manometer has recently been described in patients both with and without Graves' disease. ${ }^{3132}$ This instrument may ultimately assist in answering the question at hand.

Increasing the effective orbital volume by removing the bony walls is the most common surgical measure to relieve orbital pressure. Rapid improvement in the congestive signs and symptoms including optic neuropathy follows bony decompressive surgery for dysthyroid orbitopathy. In some cases, removal of only the lateral wall of the orbit has been documented to reverse optic neuropathy. ${ }^{10}$ In the patients we report on, reduction of the orbital soft tissue volume has the same effect. It remains to be defined how much pressure reduction is necessary and in which patients fat removal alone will be sufficient to reverse optic neuropathy. It is clear from our experience that removal of a relatively small volume of fat may be sufficient to restore optic nerve function. In the patients we present, the removal of between 4-6 $\mathrm{ml}$ of anterior and mid-orbital fat was sufficient to relieve optic neuropathy even in the cases in which the extraocular muscles were enlarged. This finding suggests that when soft tissue volume is removed or orbital volume is expanded in any compartment, critical relief of pressure at the apex may be achieved.

In cases 1 and 2, optic neuropathy may have resulted in part from optic nerve stretch. In both cases the optic nerve shadow on CT was straight. We know that sudden forward displacement of the globe causes stretching of the optic nerve that may in turn produce neuropathy, particularly if the posterior globe is tented at an angle more acute than 130 degrees. ${ }^{33}$ Traction on the optic nerve vasculature is thought to be the underlying mechanism. ${ }^{34}{ }^{35}$ Thyroid ophthalmopathy, a relatively chronic process, is not typically associated with globe tentings. ${ }^{33}$ None the less, in cases of neuropathy with straightening of the optic nerve, removal of soft tissue volume should be equally as effective at reducing proptosis thus reversing the optic neuropathy as the more traditional technique of bony expansion. ${ }^{34}$

Analysis of high resolution CT images and our clinical experience support the view that patients with dysthyroid optic neuropathy are an inhomogeneous group. The traditionally accepted mechanism of apical compression of the optic nerve by enlarged extraocular muscles may play a variably significant part in each case. It is likely that more than one mechanism contributes to the neuropathy. Reversal of the neuropathy may be achieved by partial reduction in any contributing component. Treatment should be selected which has the least morbidity and reverses the pathology as defined by preoperative imaging.

While there is a well recognised trend towards spontaneous improvement in nearly all cases of dysthyroid orbitopathy, once neuropathy has developed few would withhold treatment, anticipating spontaneous regression, as permanent visual loss usually results. Each patient in our series had sufficient clinical criteria to warrant bone decompression. In each case, fat decom- 
pression achieved the reversal of optic neuropathy that would have been expected had a bone decompression been performed successfully. It may be argued that each case was treated with radiotherapy and/or steroids and therefore the report lacks experimental purity. However, it is equally true that most patients that ultimately undergo bone decompression have also undergone one or both of these therapies. Successful results are generally attributed to the surgery. In addition, the use of fat decompression in the treatment algorithm we employ, requires failure of maximal medical treatment before the consideration of surgical options.

We consider orbital fat decompression in cases of dysthyroid optic neuropathy in which orbital radiotherapy and corticosteroids have failed or are contraindicated. At this time we believe that CT or magnetic resonance (MR) images must show enlargement of the orbital fat compartment. This may be identified by direct measurements of the orbital fat volume. Alternatively, in the presence of clinical evidence of proptosis, radiographically normal or modestly enlarged rectus muscles or straightened optic nerves provide indirect evidence of enlargement of the orbital fat compartment. We would not now routinely recommend fat decompression in those cases in which CT or MR demonstrates significantly enlarged rectus muscles obliterating the perineural space at the orbital apex.

The cases are presented to illustrate an alternative successful surgical treatment in selected cases of dysthyroid optic neuropathy that provokes reconsideration of the mechanism of disease.

Paper presented at the 26th Annual Meeting of the American Society of Ophthalmic Plastic and Reconstructive Surgery, Atlanta, GA, USA.

1 Kennerdell JS, Rosenbaum, AE, El-Hoshy MJ. Apical optic nerve compression of dysthyroid optic neuropathy on computed tomography. Arch Ophthalmol 1981;99:807-9.

2 Trokel SL, Jakobiec FA. Correlation of CT scanning and pathologic features of ophthalmic Graves' disease. Ophthalmology 1981;88:553-64.

3 Neigel JM, Rootman J, Belkin RI, et al. Dysthyroid optic neuropathy. The crowded orbital apex syndrome. Ophthalmology 1988;95:1515-21.

4 Trobe, JD, Glaser, JS, Laflamme P. Dysthyroid optic neuropathy. Arch Ophthalmol 1978;96:1199-209.

5 Day RM, Carroll FD. Corticosteroids in the treatment of optic nerve involvement associated with thyroid dysfunction. Trans Am Ophthalmol Soc 1967;65:41-51.

6 Werner SC. Prednisone in emergency treatment of malignant exophthalmos. Lancet 1966;1:1004-7.

7 Kazim M, Trokel S, Moore S. Treatment of acute Graves' Kazim M, Trokel S, Moore S. Treatment of acu

8 Prummel MF, Mouritis MP, Blank L, et al. Randomised double-blind trial of prednisone versus radiotherapy in Graves' ophthalmopathy. Lancet 1993;342:949-54.
9 Donaldson SS, Bagshaw MA, Kriss JP. Supervoltage orbital Donaldson SS, Bagshaw MA, Kriss JP. Supervoltage orbital
radiotherapy for Graves' ophthalmopathy. F Clin Endocrinol Metab 1973;37:276-85.

10 Dollinger J. Die Druckentlastung der Augenhole durch Entfernung der auBern Orbitalwand bei hochgradigem Exophthamus (Morbus Basedowii) und konsekutiver Honhauterkrankung. Dtsch Med Wochenschr 1911;37:1888-90.

11 Walsh TE, Ogura JH. Transantral orbital decompression for malignant exophthalmos. Laryngoscope 1957;67:544-68.

12 Ogura JH, Lucente FE. Surgical results of orbital decompression for malignant exophthalmos. Laryngoscope 1974; 84:637-44.

13 Leone CR, Bajandas FJ. Inferior orbital decompression for dysthyroid optic neuropathy. Ophthalmology $1981 ; 88$ :5252

14 Johnson MH. Anterior orbitotomy. Ophthalmic Surg 1979; 10:69-73.

15 Trokel S, Kazim M, Moore S. Orbital fat removal: decompression for Graves' orbitopathy. Ophthalmology 1993;100:674-82.

16 Olivari N. Transpalpebral decompression of endocrine ophthalmopathy (Graves' disease) by removal of intraorbital thalmopathy (Graves' disease) by removal of intraorbital Reconstr Surg 1991;87:627-43.

17 Day RM, Carroll FD. Optic nerve involvement associated with thyroid dysfunction. Arch Ophthalmol 1962;67:28997.

18 Char D. Thyroid eye disease. Baltimore: Williams and Wilkins 1985:101.

19 Slavin ML, Glaser JS. Acute severe irreversible visual loss with sphenoethmoiditis - "posterior" orbital cellulitis. Arch Ophthalmol 1987;105:345-8.

20 Skalka H. Perineural optic nerve changes in endocrine orbitopathy. Arch Ophthalmol 1978;96:468-473.

21 Hufnagel TJ, Hickey WF, Cobbs WH, et al. Immunohistochemical and ultrastructural studies on the exenterated orbital tissues of a patient with Graves' disease. Ophthalmology 1984;91:1411-19.

22 Forbes G, Gorman CA, Gehring D, et al. Computer analysis of orbital fat and muscle volumes in Graves' ophthalmopathy. Am f NeuroRadiol 1983;4:737-40.

23 Peyster RG, Ginsberg F, Silber JH, et al. Exophthalmos caused by excessive fat: CT volumetric analysis and differential diagnosis. Am ₹ NeuroRadiol 1986;146:459-64.

24 Forbes G, Gorman CA, Brennan MD, et al. Ophthalmology of Graves' disease: computerized volume measurements of the orbital fat and muscle. Am F NeuroRadiol 1986;7:6516.

25 Zonneveld FW, Koornneef L, Wittebol-Post D. Quantitative volumetric assessment of orbital soft tissue. In: von Lemke H, Rhodes ML, Jaffe CC, Felix R, eds. Computer assisted radiology. Berlin, Heidelberg: Springer-Verlag, 1991:181-6.

26 Nunery WR. Ophthalmic Graves' disease: a dual theory of pathogenesis. In: Nunery WR, ed. Ophthalmol Clin North Am 1991:4.

27 Trobe JD. Optic nerve involvement in dysthyroidism. Ophthalmology 1981;88:488-92.

28 Feldon, SE, Muramatsu S, Werner JM. Clinical classification of Graves' ophthalmopathy: identification of risk factors for optic neuropathy. Arch Ophthalmol 1984;102: 1469-72.

29 Freuh BR, Musch DC, Grill R, et al. Orbital compliance in Graves' eye disease. Ophthalmology 1985;92:657-65.

30 Wolfe SA. Discussion of Olivari N. Transpalpebral decompression of endocrine ophthalmopathy by removal of intraorbital fat. Plast Reconstr Surg 1991;876:42-3.

31 Riemann CD, Foster JA, Kosmorsky GS. Direct orbital manometry in patients with thyroid-associated orbitopathy. Ophthalmology 1999;106:1296-305.

32 Riemann CD, Foster JA, Kosmorsky GS. Direct orbital mannometry in healthy patients. Ophthal Plastic Recon Surg 1999;15:121-5

33 Dalley, Robertson WD, Rootman J. Globe tenting:a sign of increased orbital tension. Am F. Neuroradiol 1989;10:181-6.

34 Anderson RL, Tweeten JP, Patrinely JR, et al. Dysthyroid optic neuropathy without extraocular muscle involvement. Ophthalmic Surg 1989;20:568-74.

35 Dolman PJ, Glazer LC, Harris GJ, et al. Mechanisms of visual loss in severe proptosis. Ophthal Plastic Reconstruct Surg 1991;7:256-60. 